\title{
Test System for Emergency Lighting based on PIC Microcontroller
}

\author{
Zhao $\mathrm{Yu}^{1, \mathrm{a}^{*}}$ \\ ${ }^{1}$ Shenyang Fire Research Institute, Wenda Street 218-20 Huanggu District, Shenyang, China \\ azhaoyu@efire.cn
}

Keywords: Emergency lighting; Test system; PIC micro control units; Performance test; Discharge time.

\begin{abstract}
This paper introduces design and structure of test system of discharge time for emergency lightings based on PIC MCU, including the structure of hardware and design of software. And the communication performance and human-machine interface (HMI) of the system are elaborated. In addition, due to functional properties and extension abilities of the components in the system, this paper analyzes possibilities of functional expansion and development in the future for the system in theory.
\end{abstract}

\section{Introduction}

Emergency lighting system powered by battery as an important part in industrial and civil buildings, play a crucial role for emergency treatment and human evacuation when unexpected events occur which lead to an interruption in the power supply of environment lighting. There are many kinds of emergency lamps with a variety of classification methods: according to the power supply source of emergency state, it can be divided into self-contained type, centrally supplied type and compound self-contained type; according to working state of emergency lamps, it can be divided into maintained and non-maintained types; according to the application of emergency lamps, it can be divided into emergency lighting lamps and emergency indicating lamps. Discharge time and lighting and indicating ability in emergency state are two most important performance indexes for emergency lamps. So both the general product standard and architectural design code put forward a clear demand of emergency time. The test system based on PIC MCU introduced in this paper is used for measurement of discharge time of battery powered emergency lamps. It provides a hardware solution for mass testing of discharge time, and a technical mean for performance testing and routine inspection of emergency lamps products.

\section{Hardware Composition of Test System}

\section{Structure of the system}

As shown in Fig. 1, is the structure diagram of test system. It consists of three parts. Optical detectors with special fixtures are used for monitor the working state of emergency lamps; testing data collection module with 24 optical detectors as a subsystem transfer testing information including working state and discharge time of lamps to the main controller of system; the main controller displays working state and discharge time of all the lamps under test in real-time, and automatically records and prints measurement results by microprint when test of anyone of subsystems is completed. Besides that, the main controller can also be used for some functional operations, such as changing system testing parameters and operating programmable output contacts in it. 


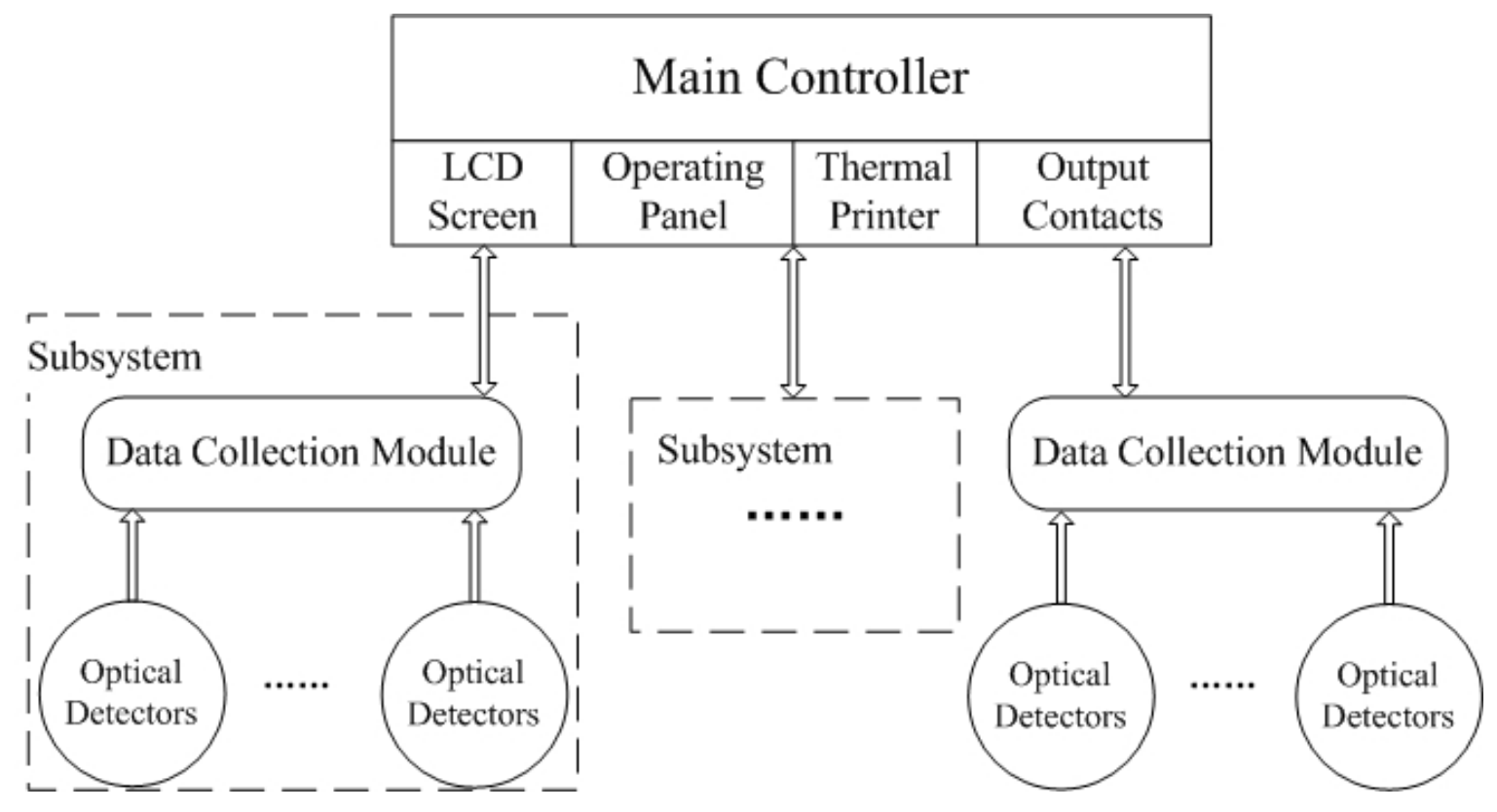

\section{Selection of optical sensor}

Fig. 1 Construction of the test system

In order to ensure accuracy of detecting, optical detectors choose industry leading optical sensor as core component, it can be used for testing all types of emergency lamps with the use of special fixture. Especially for measurement of fire emergency indicating lamps, the perfect quality of sensors embodies the superiority. Because the national standard GB 17945-2010 <Fire emergency lighting and evacuate indicating system> stipulates that at the time lamps turn into emergency state and 80 minutes after batteries start discharging, surface luminance of indicating lamps should not be less than $50 \mathrm{~cd} / \mathrm{m}^{2}$, so by setting the threshold value of sensors, the optical detectors can distinguish working states of indicating lamps at a steady range of $45 \mathrm{~cd} / \mathrm{m}^{2} \sim 48 \mathrm{~cd} / \mathrm{m}^{2}$. It is possible that when the surface luminance of lamps could not meet the requirement of the standard, detectors determine that emergency lamps has been extinguished, even if lamps is still lit but faint.

Besides that, optical detectors can also be used for testing flash indicating lamps by setting sampling frequency and criteria of optical sensors. For example, the flash fire emergency indicating lamps which meet the requirements of GB 17945-2010 and GB 7000.2-2010, whose frequency of flash is $1 \mathrm{~Hz}$, and duty ratio is not less than $80 \%$, can also be measured continuously and accurately by the test system.

\section{Selection of MCU}

Both the main controller and data collection module choose Microchip's PIC micro control unit as the main control chip. PIC MCU has perfect function, low power consumption, strong anti-interference ability, and efficient programming instruction instruction, which can not only be applicable to complex detection equipment, but also adaptable to serious working environment. The data collection module uses PIC16F1512 series 8 bit flash memory chip, and the main controller uses PIC24FJ256 series of 16 bit flash memory chip with USB OTG function. PIC16F1512 series chips are a high performance $\mathrm{MCU}$ with RISC function, it has optimized $\mathrm{C}$ compiler architecture, and the number of program instruction is only 49 , so the development process is more efficient. 25 pins out of 28 pins of the chip are the I/O ports, which make data collection module has 24 sampling channels for monitoring the state of lamps and measuring charging time. In addition, with the logic chips in the circuit, the collecting modules can also display working state of all the 24 detected lamp on the surface in real-time. While the field power supply mode, and the use of special power supply management chip in the circuit guarantee the stability of the optical detectors and main chip, improve the reliability of the collecting module. 
PIC24FJ256 series 16 bit flash memory chips adopt the improved Harvard structure, which have rich I/O resources, and provide multiple peripheral interfaces. the 16 bit chips could control display, printer, external input and output module and other external components. The working frequency of the MCU is $32 \mathrm{MHz}$, the maximum space of program is $12 \mathrm{MB}$, and it is facilitate for transplantation. In addition, these series chips have a USB OTG (On-The-Go) function, which could provide on-chip functions in accordance with the USB2.0 standard, and the realization of function application is wider and more flexible. These functions provide hardware base for function expansion and network integration for the main controller. Power management unit of the controller is a switching power supply module having UPS function, with a standby battery, which guarantee the test system also can realize data acquisition and recording even in the absence of power, and ensure reliability of test system and security of measuring data.

\section{Software Design of Test System}

\section{Program flow}

In the test system, each data collecting module has independent address for communication, host display and record data and information of test in module. When powered on, the main controller scans modules online in real-time, displays number and address of modules. Test system can also insert new modules in communication at any time when operating, to expand capacity of system. And if any module get disconnected in communication, the controller can also detect missing of module , give an alarm to users and indicate related information. Modules display working state of every emergency lamp in real-time, waiting for the test start command. The system can start the test by sending a broadcast from main controller to all the modules, and each module can also start a separate test with all the optical detectors connected as a subsystem. Whether being testing or standby, the working state of modules can also be clearly displayed on the controller. After the start of the test, data collecting modules accumulate the discharge time of emergency lamps under test respectively based on the initial input state of each channel. Any channel has no input signal at the beginning of test, modules will take the channel idle and the discharge time will always be zero even if the state of input changed. During the test, modules will upload working state information and test data of respective subsystem to the main controller, all the measurement data of system will be displayed on the screen of the controller and recorded, until a new mission of test is starting.

When the optical detectors detect any emergency lamp extinguish, modules will immediately stop the relative timer and record the discharge time as the test result of corresponding channel. And even if the lamp is lit again after that the discharge time will not be accumulated until the module restart a new test. At the same time, because the sampling frequency and the criterion of modules have been set, flash emergency lamps during discharging could also be test without any interference. When all the timers of the channels on the module stop, test mission of the subsystem is completed, and the test information will be submitted to the main controller. After getting the instruction of test ending the controller will print automatically the test results through the thermal printer and display that on the corresponding interface of the LCD screen, until the module receives a new test start command. In addition, there is a functional option of test start delay on the parameter setting interface of controller. By setting the time delay, the controller will send out the command of start after the interval. it is flexible to adjust method and procedure of test. For exmaple by connecting power supply of lamps to the time delay relay in the controller it is possible to charge the batteries of lamps before starting a test.

Test system measures the discharge time of lamps in minute with resolution for 1 minute, and the maximum range is 9999 minutes. Any channel accumulates 300 minutes, and the deviation of timer is less than 5 seconds. it meets the requirements of the product standards for the range and precision of test equipment.

\section{Communication protocol and system capacity}

The main controller and modules use the standard MODBUS communication protocol, which is stable and reliable as an industrial field bus protocol. And the maximum distance of effective communication 
between controller and the data modules is up to 500 meters. The controller and modules with RS-232 as a physical interface, whose electrical structure is not only simple and practical, it is convenient to expand capacity of the system. With a single main controller, the maximum amount of subsystems which consist of a module and 24 detectors, i.e., the maximum number of modules online can be up to 200. So the maximum capacity of test system is up to 4800 channels, which is enough to meet the requirements of test in library or routine inspection in the workplace.

\section{The Human-Machine interface}

The test system has a friendly human-machine interface. The main controller displays all of the information of system and performs all of the operations from user. The controller has LCD screen, operating panel, indicating lamp and a thermal printer on the surface, inside which there are output contacts with programmable and manual two modes of control. the screen can display data of the test from all modules online, comprising status and discharge time of each lamp under test, total number of modules online and address and working mode of each one. All the functions of system can be operated on the panel including setting parameters of system, switching the working state of modules, printing the real-time test data and controlling the output contacts manually. The indicator lights on the surface can prompt users when modules being offline and the status of output contacts changing. The thermal printer is able to print all the data of test, including date and time of the test, discharge time of lamps and other information displayed on the screen. These are not only output and record from the controller, but also can be used as a evidence of test. It is convenient and flexible to be used in different application fields.

\section{Functional Expansion of the System}

Each component in the test system has independence and compatibility in function, so it is sample to achieve functional expansion using each part of the system. For example, by connecting the power supply of lamps under test to output contacts of controller, it is possible to control charging time of batteries according to the delay time of relay. And because of the standard communication interface and protocol, it is probable to build a network of test systems, or a larger database of test. With other data processing terminals such as industial computers and programmable logic controllers, the subsystems including modules and optical detectors can build a new test system which can flexibly change construction of system and the method of data processing to meet the actual needs. Because of the rich external interfaces and perfect compatibility, it will play an important role for automation of test in the future.

\section{Conclusion}

Test system for emergency lightings gives full play to the performance of optical sensors and the PIC microcontrollers, realizes the anticipative purpose, and fills the blank in the field of test equipment for emergency lightings. The design of hardware is reliable, communication performance is reliable and the HMI interface is friendly, which ensure the data of measurement accurate and reliable. And rich external interfaces for expansion and good compatibility of system will bring more functional application in the future.

\section{References}

[1] Luminaires - Part 2-22: Particular requirements - Luminaires for emergency lighting, GB 7000.2 - 2008/IEC 60598-2-22:2002.

[2] Fire emergency lighting and evacuate indicating system, GB 17945 - 2010. 